\title{
ON FIXED POINT THEOREMS IN INTUITIONISTIC FUZZY METRIC SPACES
}

\author{
Cihangir Alaca
}

\begin{abstract}
In this paper, we give some new fixed point theorems for contractive type mappings in intuitionistic fuzzy metric spaces. We improve and generalize the well-known fixed point theorems of Banach [4] and Edelstein [8] in intuitionistic fuzzy metric spaces. Our main results are intuitionistic fuzzy version of Fang's results [10]. Further, we obtain some applications to validate our main results to product spaces.
\end{abstract}

\section{Introduction}

In 1965, the concept of fuzzy sets was introduced initially by Zadeh [28]. Since then, many authors have expansively developed the theory of fuzzy sets and applications. Especially, Deng [7], Erceg [9], Kaleva and Seikkala [14], Kramosil and Michalek [15] have introduced the concepts of fuzzy metric spaces in different ways. Mishra et al. [18] and Singh and Tomar [25] obtained some fixed point theorems and these fixed point theorems applied to product spaces.

Alaca et al. [2] using the idea of intuitionistic fuzzy sets [3, 6], they defined the notion of intuitionistic fuzzy metric space (shortly I-FM space) as Park [20] with the help of continuous t-norms and continuous t-conorms as a generalization of fuzzy metric space due to Kramosil and Michalek [15]. Further, they introduced the notion of Cauchy sequences in an I-FM spaces and proved the well-known fixed point theorems of Banach [4] and Edelstein [8] extended to I-FM spaces with the help of Grabiec [11]. Turkoglu et al. [27] introduced the concept of compatible maps and compatible maps of types $(\alpha)$ and $(\beta)$ in I-FM spaces and gave some relations between the concepts of compatible maps and compatible maps of types $(\alpha)$ and $(\beta)$. Turkoglu et al. [26] gave generazation of Jungck's common fixed point theorem [13] to I-FM spaces. They first formulate the definition of weakly commuting and R-weakly commuting mappings in I-FM spaces and proved the intuitionistic fuzzy version of Pant's theorem

Received February 8, 2006; Revised August 17, 2009.

2000 Mathematics Subject Classification. 54H25, 54A40, 47H10.

Key words and phrases. triangular norm, triangular conorm, I-FM space, contractive type mappings, fixed point. 
[19]. Many authors studied the concept of I-FM space and its applications $[1,2,12,20,21,22,23]$.

In the present paper, we give some new fixed point theorems for contractive type mappings in I-FM spaces. We improve and generalize the well-known fixed point theorems of Banach [4] and Edelstein [8] in I-FM spaces. Our main results are intuitionistic fuzzy version of Fang's results [10]. Finally, we obtain some applications to validate our main results on the product of an I-FM space.

\section{Intuitionistic fuzzy metric spaces}

Definition 1 ([24]). A binary operation $*:[0,1] \times[0,1] \rightarrow[0,1]$ is continuous t-norm if $*$ is satisfying the following conditions:

(i) $*$ is commutative and associative;

(ii) $*$ is continuous;

(iii) $a * 1=a$ for all $a \in[0,1]$;

(iv) $a * b \leq c * d$ whenever $a \leq c$ and $b \leq d$ for all $a, b, c, d \in[0,1]$.

Definition 2 ([24]). A binary operation $\diamond:[0,1] \times[0,1] \rightarrow[0,1]$ is continuous t-conorm if $\diamond$ is satisfying the following conditions:

(i) $\diamond$ is commutative and associative;

(ii) $\diamond$ is continuous;

(iii) $a \diamond 0=a$ for all $a \in[0,1]$;

(iv) $a \diamond b \leq c \diamond d$ whenever $a \leq c$ and $b \leq d$ for all $a, b, c, d \in[0,1]$.

The concepts of triangular norms (t-norms) and triangular conorms (t-conorms) are known as the axiomatic skeletons that we use for characterizing fuzzy intersections and unions, respectively. These concepts were originally introduced by Menger [17] in his study of statistical metric spaces.

The following definition and the fundamental properties of I-FM spaces due to Kramosil and Michalek [15] was given by Alaca et al. [2].

Definition $3([2])$. A 5 -tuple $(X, M, N, *, \diamond)$ is said to be an I-FM space if $X$ is an arbitrary set, $*$ is a continuous t-norm, $\diamond$ is a continuous t-conorm and $M, N$ are fuzzy sets on $X^{2} \times[0, \infty)$ satisfying the following conditions:

(i) $M(x, y, t)+N(x, y, t) \leq 1$ for all $x, y \in X$ and $t>0$;

(ii) $M(x, y, 0)=0$ for all $x, y \in X$;

(iii) $M(x, y, t)=1$ for all $x, y \in X$ and $t>0$ if and only if $x=y$;

(iv) $M(x, y, t)=M(y, x, t)$ for all $x, y \in X$ and $t>0$;

(v) $M(x, y, t) * M(y, z, s) \leq M(x, z, t+s)$ for all $x, y, z \in X$ and $s, t>0$;

(vi) for all $x, y \in X, M(x, y, \cdot):[0, \infty) \rightarrow[0,1]$ is left continuous;

(vii) $\lim _{t \rightarrow \infty} M(x, y, t)=1$ for all $x, y \in X$ and $t>0$;

(viii) $N(x, y, 0)=1$ for all $x, y \in X$;

(ix) $N(x, y, t)=0$ for all $x, y \in X$ and $t>0$ if and only if $x=y$;

(x) $N(x, y, t)=N(y, x, t)$ for all $x, y \in X$ and $t>0$;

(xi) $N(x, y, t) \diamond N(y, z, s) \geq N(x, z, t+s)$ for all $x, y, z \in X$ and $s, t>0$; 
(xii) for all $x, y \in X, N(x, y, \cdot):[0, \infty) \rightarrow[0,1]$ is right continuous;

(xiii) $\lim _{t \rightarrow \infty} N(x, y, t)=0$ for all $x, y$ in $X$.

Then $(M, N)$ is called an intuitionistic fuzzy metric on $X$. The functions $M(x, y, t)$ and $N(x, y, t)$ denote the degree of nearness and the degree of nonnearness between $x$ and $y$ with respect to $t$, respectively.

Remark 1. Every fuzzy metric space $(X, M, *)$ is an I-FM space of the form $(X, M, 1-M, *, \diamond)$ such that t-norm $*$ and t-conorm $\diamond$ are associated $([16])$, i.e., $x \diamond y=1-((1-x) *(1-y))$ for all $x, y \in X$.

Remark 2. In I-FM space $X, M(x, y, \cdot)$ is non-decreasing and $N(x, y, \cdot)$ is nonincreasing for all $x, y \in X$.

Definition $4([2])$. Let $(X, M, N, *, \diamond)$ be an I-FM space. Then

(i) a sequence $\left\{x_{n}\right\}$ in $X$ is said to be Cauchy sequence if, for all $t>0$ and $p>0$,

$$
\lim _{n \rightarrow \infty} M\left(x_{n+p}, x_{n}, t\right)=1, \quad \lim _{n \rightarrow \infty} N\left(x_{n+p}, x_{n}, t\right)=0 .
$$

(ii) a sequence $\left\{x_{n}\right\}$ in $X$ is said to be convergent to a point $x \in X$ if, for all $t>0$,

$$
\lim _{n \rightarrow \infty} M\left(x_{n}, x, t\right)=1, \quad \lim _{n \rightarrow \infty} N\left(x_{n}, x, t\right)=0 .
$$

Since $*$ and $\diamond$ are continuous, the limit is uniquely determined from $(v)$ and (xi), respectively.

Definition $5([2])$. An I-FM space $(X, M, N, *, \diamond)$ is said to be complete if and only if every Cauchy sequence in $X$ is convergent.

Definition $6([2])$. An I-FM space $(X, M, N, *, \diamond)$ is said to be compact if every sequence in $X$ contains a convergent subsequence.

Lemma 1 ([2]). (i) If $\lim _{n \rightarrow \infty} x_{n}=x$ and $\lim _{n \rightarrow \infty} y_{n}=y$, then,

$$
M(x, y, t) \leq \lim _{n \rightarrow \infty} \inf M\left(x_{n}, y_{n}, t\right) \text { and } N(x, y, t) \geq \lim _{n \rightarrow \infty} \sup N\left(x_{n}, y_{n}, t\right)
$$

for all $t>0$.

(ii) If $\lim _{n \rightarrow \infty} x_{n}=x$ and $\lim _{n \rightarrow \infty} y_{n}=y$, then,

$$
M(x, y, t) \geq \lim _{n \rightarrow \infty} \sup M\left(x_{n}, y_{n}, t\right) \text { and } N(x, y, t) \leq \lim _{n \rightarrow \infty} \inf N\left(x_{n}, y_{n}, t\right)
$$

for all $t>0$.

Particularly, if $M(x, y, \cdot)$ is continuous at point $t$, then

$$
\lim _{n \rightarrow \infty} M\left(x_{n}, y_{n}, t\right)=M(x, y, t) \text { and } \lim _{n \rightarrow \infty} N\left(x_{n}, y_{n}, t\right)=N(x, y, t) .
$$

The following two theorems extend the well-known fixed point theorems of Banach [4] and Edelstein [8] to I-FM spaces in the sense of Kramosil and Michalek [15] was given by Alaca et al. [2]. 
Theorem $1([2])$. Let $(X, M, N, *, \diamond)$ be a complete I-FM space. Let $T: X \rightarrow$ $X$ be a mapping satisfying

$$
M(T x, T y, k t) \geq M(x, y, t) \text { and } N(T x, T y, k t) \leq N(x, y, t)
$$

for all $x, y$ in $X, 0<k<1$. Then $T$ has a unique fixed point.

Theorem $2([2])$. Let $(X, M, N, *, \diamond)$ be a compact space. Let $T: X \rightarrow X$ be a mapping satisfying

$$
M(T x, T y, \cdot)>M(x, y, \cdot) \text { and } N(T x, T y, \cdot)<N(x, y, \cdot)
$$

for all $x \neq y$.

$$
\left(\begin{array}{c}
\text { i.e., } M(T x, T y, \cdot) \geq M(x, y, \cdot) \text { and } M(T x, T y, \cdot) \neq M(x, y, \cdot) \\
N(T x, T y, \cdot) \leq N(x, y, \cdot) \text { and } N(T x, T y, \cdot) \neq N(x, y, \cdot) \text { for all } x \neq y .
\end{array}\right)
$$

Then $T$ has a unique fixed point.

\section{Main results}

Lemma 2. Let $*$ be a continuous t-norm and $\diamond$ be a continuous t-conorm. Then for each $\lambda \in(0,1)$, there is a sequence $\left\{\lambda_{n}\right\}$ in $(0,1)$ such that

$$
\left(1-\lambda_{n}\right) *\left(1-\lambda_{n}\right)>1-\lambda_{n-1} \text { and } \lambda_{n} \diamond \lambda_{n}<\lambda_{n-1}, \quad n=1,2, \ldots
$$

where $\lambda_{0}=\lambda$ (obviously, the sequence $\left\{\lambda_{n}\right\}$ satisfying condition (3.1) is decreasing).

Proof. Since $*$ is continuous at point Definition 3 [(vii) and (xiii)] and $a * b \leq$ $1 * 1=1$ and $(1-a) \diamond(1-a) \geq 0 \diamond 0=0$ for all $a, b \in[0,1]$, we get

$$
\sup _{0<\mu<1}[(1-\mu) *(1-\mu)]=1, \inf _{0<\mu<1}[\mu * \mu]=0 .
$$

Hence, for each $\lambda \in(0,1)$, there exists $\lambda_{1} \in(0,1)$ such that

$$
\left(1-\lambda_{1}\right) *\left(1-\lambda_{1}\right)>1-\lambda \text { and } \lambda_{1} \diamond \lambda_{1}<\lambda .
$$

Similarly, from (3.2) there exists $\lambda_{2} \in(0,1)$ such that

$$
\left(1-\lambda_{2}\right) *\left(1-\lambda_{2}\right)>1-\lambda_{1} \text { and } \lambda_{2} \diamond \lambda_{2}<\lambda_{1} \text {. }
$$

Continuing this procedure we can obtain a sequence $\left\{\lambda_{n}\right\} \subset(0,1)$ satisfying condition (3.1). This completes the proof.

Lemma $3([5])$. Let the function $\phi(t):[0, \infty) \rightarrow[0, \infty)$ satisfying the following condition:

$\left(\phi_{1}\right) \phi(t)$ is strictly increasing, $\phi(0)=0$ and $\lim _{n \rightarrow \infty} \phi^{n}(t)=\infty$ for all $t>0$, where $\phi^{n}(t)$ denotes the $n$-th iterative function of $\phi(t)$. Then $\phi(t)>t, \phi^{n}(t)>$ $\phi^{n-1}(t), \forall t>0, n=1,2, \ldots$ 
Lemma 4. Let $(X, M, N, *, \diamond)$ be an $I-F M$ space. Let $T: X \rightarrow X$ be a mapping satisfying

$$
M\left(T x, T y, t_{1}\right)>M\left(x, y, t_{1}\right) \text { and } N\left(T x, T y, t_{1}\right)<N\left(x, y, t_{1}\right)
$$

where $t_{1}$ is a fixed positive number. Then there exists a continuity point $t_{0}$ of $M(x, y, \cdot)$ such that

$$
M\left(T x, T y, t_{0}\right)>M\left(x, y, t_{0}\right) \text { and } N\left(T x, T y, t_{0}\right)<N\left(x, y, t_{0}\right) .
$$

Proof. Since $M(T x, T y, \cdot)-M(x, y, \cdot)$ and $N(T x, T y, \cdot)-N(x, y, \cdot)$ are leftcontinuous and right-continuous, respectively, at point $t_{1}$, by (3.3) there exists $0<t_{2}<t_{1}$ such that

$$
M(T x, T y, t)>M(x, y, t) \text { and } N(T x, T y, t)<N(x, y, t)
$$

for all $t \in\left[t_{2}, t_{1}\right]$. Note that the set of discontinuous points of $M(x, y, \cdot)$ and $N(x, y, \cdot)$ are countable at most. Thus, there exists $t_{0} \in\left[t_{2}, t_{1}\right]$ such that $M(x, y, \cdot)$ and $N(x, y, \cdot)$ are continuous at $t_{0}$. Thus (3.4) holds. This completes the proof.

Theorem 3. Let $(X, M, N, *, \diamond)$ be a complete $I-F M$ space. Let $T: X \rightarrow X$ be a mapping satisfying the following conditions:

(i) there exists $x_{0} \in X$ such that

$$
\lim _{t \rightarrow \infty} M\left(x_{0}, T^{i} x_{0}, t\right)=1 \text { and } \lim _{t \rightarrow \infty} N\left(x_{0}, T^{i} x_{0}, t\right)=0, i=1,2, \ldots ;
$$

(ii) there exists a mapping $m: X \rightarrow \mathbb{N}$ such that for any $x, y$ in $X$,

$$
\begin{aligned}
M\left(T^{m(x)} x, T^{m(x)} y, t\right) & \geq M(x, y, \phi(t)) \text { and } \\
N\left(T^{m(x)} x, T^{m(x)} y, t\right) & \leq N(x, y, \phi(t)),
\end{aligned}
$$

where the function $\phi(t)$ satisfies condition $\left(\phi_{1}\right)$ and

$\left(\phi_{2}\right) \lim _{t \rightarrow \infty}[\phi(t)-t]=\infty$.

Then $T$ has a unique fixed point $x_{*}$, and the quasi-iterative sequence $\left\{x_{n}\right.$ : $\left.T^{m\left(x_{n-1}\right)} x_{n-1}\right\}$ converges to $x_{*}$.

Proof. First we prove that

$$
\sup _{s>0} \inf _{x \in O_{T}\left(x_{0}\right)} M\left(x_{0}, x, s\right)=1 \text { and } \inf _{s>0} \sup _{x \in O_{T}\left(x_{0}\right)} N\left(x_{0}, x, s\right)=0
$$

where $O_{T}\left(x_{0}\right)=\left\{x_{0}, T x_{0}, T^{2} x_{0}, \ldots\right\}$ is called the orbit of $x_{0}$ for $T$. For any $n \in \mathbb{N}$ with $n>m\left(x_{0}\right)$, we can denote

$$
n=k m\left(x_{0}\right)+s, \text { where } 0 \leq s<m\left(x_{0}\right) .
$$

Note that $\phi(t)>t$ for all $t>0$ and $\lim _{t \rightarrow \infty}[\phi(t)-t]=\infty$. By $(3.5)$, we have

$$
\lim _{t \rightarrow \infty} M\left(x_{0}, T^{i} x_{0}, \phi(t)\right)=1 \text { and } \lim _{t \rightarrow \infty} N\left(x_{0}, T^{i} x_{0}, \phi(t)\right)=0
$$


for $i=1,2, \ldots, m\left(x_{0}\right)$ and

(3.9) $\lim _{t \rightarrow \infty} M\left(x_{0}, T^{m\left(x_{0}\right)} x_{0}, \phi(t)-t\right)=1$ and $\lim _{t \rightarrow \infty} N\left(x_{0}, T^{m\left(x_{0}\right)} x_{0}, \phi(t)-t\right)=0$.

Moreover, from Lemma 2 , for any $\lambda \in(0,1)$, there exists a sequence $\left\{\lambda_{n}\right\}$ in $(0,1)$ such that

$$
\left(1-\lambda_{n}\right) *\left(1-\lambda_{n}\right)>1-\lambda_{n-1} \text { and } \lambda_{n} \diamond \lambda_{n}<\lambda_{n-1}, \quad\left(\lambda_{0}=\lambda\right) n=1,2, \ldots
$$

Thus, it follows from (3.8) and (3.9) that for given $\lambda_{k}$ there exists $t_{0}>0$ such that

$$
\min _{1 \leq i \leq m\left(x_{0}\right)} M\left(x_{0}, T^{i} x_{0}, \phi(t)\right)>1-\lambda_{k} \text { and } \max _{1 \leq i \leq m\left(x_{0}\right)} N\left(x_{0}, T^{i} x_{0}, \phi(t)\right)<\lambda_{k},
$$

and

$M\left(x_{0}, T^{m\left(x_{0}\right)} x_{0}, \phi(t)-t\right)>1-\lambda_{k}$ and $N\left(x_{0}, T^{m\left(x_{0}\right)} x_{0}, \phi(t)-t\right)<\lambda_{k}, \forall t>t_{0}$.

Thus, from (3.6), we get

$$
\begin{aligned}
& M\left(x_{0}, T^{n} x_{0}, \phi(t)\right) \\
= & M\left(x_{0}, T^{k m\left(x_{0}\right)+s} x_{0}, \phi(t)\right) \\
\geq & M\left(x_{0}, T^{m\left(x_{0}\right)} x_{0}, \phi(t)-t\right) * M\left(T^{m\left(x_{0}\right)} x_{0}, T^{k m\left(x_{0}\right)+s} x_{0}, t\right) \\
\geq & M\left(x_{0}, T^{m\left(x_{0}\right)} x_{0}, \phi(t)-t\right) * M\left(x_{0}, T^{(k-1) m\left(x_{0}\right)+s} x_{0}, \phi(t)\right) \geq \cdots \\
\geq & M\left(x_{0}, T^{m\left(x_{0}\right)} x_{0}, \phi(t)-t\right) * \cdots * M\left(x_{0}, T^{m\left(x_{0}\right)} x_{0}, \phi(t)-t\right) \\
& * M\left(x_{0}, T^{s} x_{0}, \phi(t)\right) \\
> & \left(1-\lambda_{k}\right) * \cdots *\left(1-\lambda_{k}\right)>\left(1-\lambda_{k-1}\right) * \cdots *\left(1-\lambda_{k-1}\right) \\
> & \cdots>\left(1-\lambda_{1}\right) *\left(1-\lambda_{1}\right)>1-\lambda, \forall t>t_{0},
\end{aligned}
$$

and

$$
\begin{aligned}
& N\left(x_{0}, T^{n} x_{0}, \phi(t)\right) \\
& =N\left(x_{0}, T^{k m\left(x_{0}\right)+s} x_{0}, \phi(t)\right) \\
& \leq N\left(x_{0}, T^{m\left(x_{0}\right)} x_{0}, \phi(t)-t\right) \diamond N\left(T^{m\left(x_{0}\right)} x_{0}, T^{k m\left(x_{0}\right)+s} x_{0}, t\right) \\
& \leq N\left(x_{0}, T^{m\left(x_{0}\right)} x_{0}, \phi(t)-t\right) \diamond N\left(x_{0}, T^{(k-1) m\left(x_{0}\right)+s} x_{0}, \phi(t)\right) \leq \cdots \\
& \leq N\left(x_{0}, T^{m\left(x_{0}\right)} x_{0}, \phi(t)-t\right) \diamond \stackrel{(k)}{\cdots} \diamond N\left(x_{0}, T^{m\left(x_{0}\right)} x_{0}, \phi(t)-t\right) \\
& \diamond N\left(x_{0}, T^{s} x_{0}, \phi(t)\right) \\
& <\lambda_{k} \diamond \cdots \diamond \lambda_{k}<\lambda_{k-1} \diamond \stackrel{(k)}{*} \cdot \diamond \lambda_{k-1} \\
& <\cdots<\lambda_{1} \diamond \lambda_{1}<\lambda, \forall t>t_{0} .
\end{aligned}
$$

Therefore

$$
\inf _{x \in O_{T}\left(x_{0}\right)} M\left(x_{0}, x, \phi(t)\right) \geq 1-\lambda \text { and } \sup _{x \in O_{T}\left(x_{0}\right)} N\left(x_{0}, x, \phi(t)\right) \leq \lambda, \forall t>t_{0} .
$$


Hence

$$
\sup _{s>0} \inf _{x \in O_{T}\left(x_{0}\right)} M\left(x_{0}, x, s\right) \geq 1-\lambda \text { and } \inf _{s>0} \sup _{x \in O_{T}\left(x_{0}\right)} N\left(x_{0}, x, s\right) \leq \lambda .
$$

By the arbitrariness of $\lambda$, we have

$$
\sup _{s>0} \inf _{x \in O_{T}\left(x_{0}\right)} M\left(x_{0}, x, s\right)=1 \text { and } \inf _{s>0} \sup _{x \in O_{T}\left(x_{0}\right)} N\left(x_{0}, x, s\right)=0 .
$$

Next, we prove that the quasi-iterative sequence $\left\{x_{n}=T^{m\left(x_{n-1}\right)} x_{n-1}\right\}_{n=1}^{\infty}$ is a Cauchy sequence. For convenience, put $m_{i}=m\left(x_{i}\right), i=0,1, \ldots$ Then by (3.5),

$$
\begin{aligned}
M\left(x_{n}, x_{n+p}, t\right) & =M\left(T^{m_{n-1}} x_{n-1}, T^{m_{n+p-1}+m_{n+p-2}+\cdots+m_{n-1}} x_{n-1}, t\right) \\
& \geq M\left(x_{n-1}, T^{m_{n+p-1}+m_{n+p-2}+\cdots+m_{n}} x_{n-1}, \phi(t)\right) \\
& \geq M\left(x_{n-2}, T^{m_{n+p-1}+m_{n+p-2}+\cdots+m_{n}} x_{n-2}, \phi^{2}(t)\right) \\
& \geq \cdots \geq M\left(x_{0}, T^{m_{n+p-1}+m_{n+p-2}+\cdots+m_{n}} x_{0}, \phi^{n}(t)\right) \\
& \geq \inf _{x \in O_{T}\left(x_{0}\right)} M\left(x_{0}, x, \phi^{n}(t)\right) \\
& \geq \sup _{0<s>\phi^{n}(t)} \inf _{T} M\left(x_{0}, x, s\right), \quad \forall t>0,
\end{aligned}
$$

and

$$
\begin{aligned}
N\left(x_{n}, x_{n+p}, t\right) & =N\left(T^{m_{n-1}} x_{n-1}, T^{m_{n+p-1}+m_{n+p-2}+\cdots+m_{n-1}} x_{n-1}, t\right) \\
& \leq N\left(x_{n-1}, T^{m_{n+p-1}+m_{n+p-2}+\cdots+m_{n}} x_{n-1}, \phi(t)\right) \\
& \leq N\left(x_{n-2}, T^{m_{n+p-1}+m_{n+p-2}+\cdots+m_{n}} x_{n-2}, \phi^{2}(t)\right) \\
& \leq \cdots \leq N\left(x_{0}, T^{m_{n+p-1}+m_{n+p-2}+\cdots+m_{n}} x_{0}, \phi^{n}(t)\right) \\
& \leq \sup _{x \in O_{T}\left(x_{0}\right)} N\left(x_{0}, x, \phi^{n}(t)\right) \\
& \leq \inf _{0<s>\phi^{n}(t)} \sup _{x \in O_{T}\left(x_{0}\right)} N\left(x_{0}, x, s\right), \quad \forall t>0 .
\end{aligned}
$$

Then by condition $\left(\phi_{1}\right)$ and (3.7) we have

$$
\lim _{n \rightarrow \infty} M\left(x_{n+p}, x_{n}, t\right)=1, \quad \lim _{n \rightarrow \infty} N\left(x_{n+p}, x_{n}, t\right)=0, \quad \forall t>0 .
$$

This means that $\left\{x_{n}\right\}$ is a Cauchy sequence in $X$. By the completeness of $X$, there exists $\lim _{n \rightarrow \infty} x_{n}=x_{*} \in X$.

Now we prove that $x_{*}$ is the unique fixed point of $T^{m_{*}}$, where $m_{*}=m\left(x_{*}\right)$. By Definition $3[(\mathrm{v})$ and (xi)] and (3.6), we have

$$
M\left(x_{*}, T^{m_{*}} x_{*}, t\right) \geq M\left(x_{*}, T^{m_{*}} x_{n}, \frac{t}{2}\right) * M\left(T^{m_{*}} x_{n}, T^{m_{*}} x_{*}, \frac{t}{2}\right)
$$

and

$$
N\left(x_{*}, T^{m_{*}} x_{*}, t\right) \leq N\left(x_{*}, T^{m_{*}} x_{n}, \frac{t}{2}\right) \diamond N\left(T^{m_{*}} x_{n}, T^{m_{*}} x_{*}, \frac{t}{2}\right)
$$


Then

$$
\begin{aligned}
& M\left(x_{*}, T^{m_{*}} x_{*}, t\right) \geq M\left(x_{*}, T^{m_{*}} x_{n}, \frac{t}{2}\right) * M\left(x_{n}, x_{*}, \phi\left(\frac{t}{2}\right)\right), \\
& N\left(x_{*}, T^{m_{*}} x_{*}, t\right) \leq N\left(x_{*}, T^{m_{*}} x_{n}, \frac{t}{2}\right) \diamond N\left(x_{n}, x_{*}, \phi\left(\frac{t}{2}\right)\right) .
\end{aligned}
$$

It is easy to prove that

$$
\lim _{n \rightarrow \infty} M\left(x_{*}, T^{m_{*}} x_{n}, u\right)=1 \text { and } \lim _{n \rightarrow \infty} N\left(x_{*}, T^{m_{*}} x_{n}, u\right)=0, \forall u>0 .
$$

In fact,

$$
\begin{aligned}
M\left(x_{*}, T^{m_{*}} x_{n}, u\right) & \geq M\left(x_{*}, x_{n}, \frac{1}{2} u\right) * M\left(x_{n}, T^{m_{*}} x_{n}, \frac{1}{2} u\right) \\
& =M\left(x_{*}, x_{n}, \frac{1}{2} u\right) * M\left(T^{m_{n-1}} x_{n-1}, T^{m_{n-1+m}} x_{n-1}, \frac{1}{2} u\right) \\
& \geq M\left(x_{*}, x_{n}, \frac{1}{2} u\right) * M\left(x_{n-1}, T^{m_{*}} x_{n-1}, \phi\left(\frac{1}{2} u\right)\right) \geq \cdots \\
& \geq M\left(x_{*}, x_{n}, \frac{1}{2} u\right) * M\left(x_{n-1}, T^{m_{*}} x_{n-1}, \phi^{n}\left(\frac{1}{2} u\right)\right) \rightarrow 1
\end{aligned}
$$

and

$$
\begin{aligned}
N\left(x_{*}, T^{m_{*}} x_{n}, u\right) & \leq N\left(x_{*}, x_{n}, \frac{1}{2} u\right) \diamond N\left(x_{n}, T^{m_{*}} x_{n}, \frac{1}{2} u\right) \\
& =N\left(x_{*}, x_{n}, \frac{1}{2} u\right) \diamond N\left(T^{m_{n-1}} x_{n-1}, T^{m_{n-1+m_{*}}} x_{n-1}, \frac{1}{2} u\right) \\
& \leq N\left(x_{*}, x_{n}, \frac{1}{2} u\right) \diamond N\left(x_{n-1}, T^{m_{*}} x_{n-1}, \phi\left(\frac{1}{2} u\right)\right) \leq \cdots \\
& \leq N\left(x_{*}, x_{n}, \frac{1}{2} u\right) \diamond N\left(x_{n-1}, T^{m_{*}} x_{n-1}, \phi^{n}\left(\frac{1}{2} u\right)\right) \rightarrow 0
\end{aligned}
$$

for $n \rightarrow \infty$. Then, letting $n \rightarrow \infty$ on the right side of (3.10), and noting the continuity of $*$ and $\diamond$ we have

$$
M\left(x_{*}, T^{m_{*}} x_{*}, t\right)=1 \text { and } N\left(x_{*}, T^{m_{*}} x_{*}, t\right)=0, \forall t>0 .
$$

This implies that $T^{m_{*}} x_{*}=x_{*}$, i.e., $x_{*}$ is a fixed point of $T^{m\left(x_{*}\right)}$. To show uniqueness, assume that $T^{m\left(x_{*}\right)} y=y$ for $y \in X$. Then

$$
M\left(x_{*}, y, t\right)=M\left(T^{m\left(x_{*}\right)} x_{*}, T^{m\left(x_{*}\right)} y, t\right) \geq M\left(x_{*}, y, \phi(t)\right)
$$

and

$$
N\left(x_{*}, y, t\right)=N\left(T^{m\left(x_{*}\right)} x_{*}, T^{m\left(x_{*}\right)} y, t\right) \leq N\left(x_{*}, y, \phi(t)\right) .
$$

On the other hand, as $M\left(x_{*}, y, t\right)$ is non-decreasing and $N\left(x_{*}, y, t\right)$ is nonincreasing, we have

$$
M\left(x_{*}, y, t\right) \leq M\left(x_{*}, y, \phi(t)\right) \text { and } N\left(x_{*}, y, t\right) \geq N\left(x_{*}, y, \phi(t)\right) .
$$


Hence

$$
M\left(x_{*}, y, t\right)=M\left(x_{*}, y, \phi(t)\right)=M\left(x_{*}, y, \phi^{n}(t)\right), \forall t>0
$$

and

$$
N\left(x_{*}, y, t\right)=N\left(x_{*}, y, \phi(t)\right)=N\left(x_{*}, y, \phi^{n}(t)\right), \forall t>0 .
$$

By the condition $\left(\phi_{1}\right)$,

$$
M\left(x_{*}, y, t\right)=1 \text { and } N\left(x_{*}, y, t\right)=0, \forall t>0 .
$$

Then by Definition 3 [(iii) and (ix)] we have $x_{*}=y$.

Finally, we prove that $x_{*}$ is unique fixed point of $T$, too. In fact, since $T^{m\left(x_{*}\right)} x_{*}=x_{*}$, it follows that $T x_{*}=T\left(T^{m\left(x_{*}\right)} x_{*}\right)=T^{m_{*}}\left(T x_{*}\right)$. Hence, $T x_{*}=x_{*}$.

Uniqueness is obvious. This completes the proof.

Corollary 1. Let $(X, M, N, *, \diamond)$ be a complete $I-F M$ space. Let $T: X \rightarrow X$ be a mapping satisfying the following conditions:

(i) there exists $x_{0} \in X$ such that

$$
\lim _{t \rightarrow \infty} M\left(x_{0}, T^{i} x_{0}, t\right)=1 \text { and } \lim _{t \rightarrow \infty} N\left(x_{0}, T^{i} x_{0}, t\right)=0, i=1,2, \ldots ;
$$

(ii) there exists a mapping $m: X \rightarrow \mathbb{N}$ such that for any $x, y$ in $X$,

$$
M\left(T^{m(x)} x, T^{m(x)} y, t\right) \geq M\left(x, y, \frac{t}{k}\right) \text { and } N\left(T^{m(x)} x, T^{m(x)} y, t\right) \leq N\left(x, y, \frac{t}{k}\right),
$$

where $0<k<1$.

Then the conclusion of Theorem 3 remains true.

Proof. Taking $\phi(t)=\frac{t}{k}$. Obviously, $\phi(t)$ satisfies the conditions $\left(\phi_{1}\right)$ and $\left(\phi_{2}\right)$. Therefore the conclusion follows from Theorem 3 directly.

Corollary 2. Let $(X, M, N, *, \diamond)$ be a complete $I-F M$ space. Let $T: X \rightarrow X$ be a mapping. If there exists a mapping $m: X \rightarrow \mathbb{N}$ such that for any $x, y$ in $X$,

$$
\begin{aligned}
M\left(T^{m(x)} x, T^{m(x)} y, t\right) & \geq M(x, y, \phi(t)) \text { and } N\left(T^{m(x)} x, T^{m(x)} y, t\right) \\
& \leq N(x, y, \phi(t)),
\end{aligned}
$$

where the function $\phi(t)$ satisfies conditions $\left(\phi_{1}\right)$ and $\left(\phi_{2}\right)$. Then $T$ has a unique fixed point $x_{*}$, and the iterative sequence $\left\{T^{n} x\right\}$ converges to $x_{*}$ for every $x \in$ $X$.

Proof. From Theorem 3, we need only to show that the iterative sequence $\left\{T^{n} x\right\}$ converges to $x_{*}$. For any $n \in \mathbb{N}$ with $n>m\left(x_{*}\right)$,

$$
n=k m\left(x_{*}\right)+s, \quad 0 \leq s<x_{*} .
$$


Since

$$
\begin{aligned}
M\left(x_{*}, T^{n} x, t\right) & =M\left(T^{m\left(x_{*}\right)} x_{*}, T^{k m\left(x_{*}\right)+s} x, t\right) \\
& \geq M\left(x_{*}, T^{(k-1) m\left(x_{*}\right)+s} x, \phi(t)\right) \\
& \geq \cdots \geq M\left(x_{*}, T^{s} x, \phi^{k}(t)\right) \rightarrow 1
\end{aligned}
$$

and

$$
\begin{aligned}
N\left(x_{*}, T^{n} x, t\right) & =N\left(T^{m\left(x_{*}\right)} x_{*}, T^{k m\left(x_{*}\right)+s} x, t\right) \\
& \leq N\left(x_{*}, T^{(k-1) m\left(x_{*}\right)+s} x, \phi(t)\right) \\
& \leq \cdots \leq N\left(x_{*}, T^{s} x, \phi^{k}(t)\right) \rightarrow 0
\end{aligned}
$$

for $n \rightarrow \infty$. It follows that

$$
\lim _{n \rightarrow \infty} M\left(x_{*}, T^{n} x, t\right)=1 \text { and } \lim _{n \rightarrow \infty} N\left(x_{*}, T^{n} x, t\right)=0, \forall t>0 .
$$

Then we get $\lim _{n \rightarrow \infty} T^{n} x=x_{*}$. This completes the proof.

Remark 3. Taking $\phi(t)=\frac{t}{k}(0<k<1)$ and $m(x) \equiv 1$ in Corollary 2, we at once obtain Theorem 1. Hence Theorem 1 is a special case of Corollary 2 .

Theorem 4. Let $(X, M, N, *, \diamond)$ be a complete $I-F M$ space with $t * t \geq t$ and $(1-t) \diamond(1-t) \leq(1-t)$ for all $t \in[0,1]$, and $T: X \rightarrow X$ be a continuous mapping satisfying

$$
\begin{gathered}
M(T x, T y, \cdot)>M(x, T x, \cdot) * M(y, T y, \cdot) * M(x, y, \cdot), \\
N(T x, T y, \cdot)<N(x, T x, \cdot) \diamond N(y, T y, \cdot) \diamond N(x, y, \cdot)
\end{gathered}
$$

for all $x \neq y$. If there exists $x_{0} \in X$ such that $\left\{T^{n} x_{0}\right\}_{n=0}^{\infty}$ has an accumulation point $x_{*} \in X$, and

$$
\begin{aligned}
& M\left(T^{n-1} x_{0}, T^{n} x_{0}, t\right) \leq M\left(T^{n} x_{0}, T^{n+1} x_{0}, t\right), \\
& N\left(T^{n-1} x_{0}, T^{n} x_{0}, t\right) \geq N\left(T^{n} x_{0}, T^{n+1} x_{0}, t\right), \forall t>0, n=1,2, \ldots,
\end{aligned}
$$

then $x_{*}$ is the unique fixed point of $T$, and $\lim _{n \rightarrow \infty} T^{n} x_{0}=x_{*}$.

Proof. Assume $T^{n} x_{0} \neq T^{n+1} x_{0}$ for each $n \in \mathbb{N}$. (If not, there is $n_{0} \in \mathbb{N}$ such that $T^{n_{0}} x_{0} \neq T^{n_{0}+1} x_{0}$. This means that $x_{*}=T^{n_{0}} x_{0}$ is a fixed point of $T$, and $\left.\lim _{n \rightarrow \infty} T^{n} x_{0}=x_{*}\right)$. Since $\left\{T^{n} x_{0}\right\}_{n=0}^{\infty}$ has an accumulation point $x_{*} \in X$, there exists a subsequence $\left\{T^{n_{i}} x_{0}\right\}, \lim _{i} T^{n_{i}} x_{0}=x_{*} .\left\{M\left(T^{n} x_{0}, T^{n+1} x_{0}, t\right)\right\}$ is non-decreasing and bounded and $\left\{N\left(T^{n} x_{0}, T^{n+1} x_{0}, t\right)\right\}$ is non-increasing and bounded. Thus, we have

$$
\begin{aligned}
& \left\{M\left(T^{n_{i}} x_{0}, T^{n_{i}+1} x_{0}, t\right)\right\} \text { and }\left\{N\left(T^{n_{i}} x_{0}, T^{n_{i}+1} x_{0}, t\right)\right\}, \\
& \left\{M\left(T^{n_{i}+1} x_{0}, T^{n_{i}+2} x_{0}, t\right)\right\} \text { and }\left\{N\left(T^{n_{i}+1} x_{0}, T^{n_{i}+2} x_{0}, t\right)\right\}
\end{aligned}
$$


are convergent to a common limit, i.e.,

$$
\begin{aligned}
\lim _{i} M\left(T^{n_{i}} x_{0}, T^{n_{i}+1} x_{0}, t\right) & =\lim _{i} M\left(T^{n_{i}+1} x_{0}, T^{n_{i}+2} x_{0}, t\right), \\
\lim _{i} N\left(T^{n_{i}} x_{0}, T^{n_{i}+1} x_{0}, t\right) & =\lim _{i} N\left(T^{n_{i}+1} x_{0}, T^{n_{i}+2} x_{0}, t\right), \forall t>0 .
\end{aligned}
$$

By the continuity of $T$, we have

$$
\lim _{i} T^{n_{i}+1} x_{0}=\lim _{i} T\left(T^{n_{i}} x_{0}\right)=T x_{*} .
$$

Suppose $T x_{*} \neq x_{*}$. Putting $y=T x$ in (3.11), we have

$$
M(x, T x, \cdot)<M\left(T x, T^{2} x, \cdot\right) \text { and } N(x, T x, \cdot)>N\left(T x, T^{2} x, \cdot\right)
$$

for every $x \neq T x$.

So by Lemma 4 , there exists a continuous point $t_{0}$ of $M\left(x_{*}, T x_{*}, \cdot\right)$ and $N\left(x_{*}, T x_{*}, \cdot\right)$ such that $M\left(T x_{*}, T^{2} x_{*}, \cdot\right)>M\left(x_{*}, T x_{*}, t_{0}\right)$ and $N\left(T x_{*}, T^{2} x_{*}, \cdot\right)$ $<N\left(x_{*}, T x_{*}, t_{0}\right)$. On the other hand, from Lemma 1 ,

$$
\begin{aligned}
M\left(x_{*}, T x_{*}, t_{0}\right) & =\lim _{i} M\left(T^{n_{i}} x_{0}, T\left(T^{n_{i}} x_{0}\right), t_{0}\right) \\
& =\lim _{i} M\left(T^{n_{i}+1} x_{0}, T^{n_{i}+2} x_{0}, t_{0}\right) \\
& \geq M\left(T x_{*}, T^{2} x_{*}, t_{0}\right)
\end{aligned}
$$

and

$$
\begin{aligned}
N\left(x_{*}, T x_{*}, t_{0}\right) & =\lim _{i} N\left(T^{n_{i}} x_{0}, T\left(T^{n_{i}} x_{0}\right), t_{0}\right) \\
& =\lim _{i} N\left(T^{n_{i}+1} x_{0}, T^{n_{i}+2} x_{0}, t_{0}\right) \\
& \leq N\left(T x_{*}, T^{2} x_{*}, t_{0}\right),
\end{aligned}
$$

a contradiction. Therefore $T x_{*}=x_{*}$, i.e., $x_{*}$ is a fixed point of $T$. Uniqueness follows at once from (3.11).

Finally, we prove that $\lim _{n \rightarrow \infty} T^{n} x_{0}=x_{*}$. Since $\lim _{i} T^{n_{i}} x_{0}=x_{*}$ and $\lim _{i} T^{n_{i}+1} x_{0}$ $=T x_{*}=x_{*}$, by Lemma 1 ,

$$
\lim _{i} \inf M\left(T^{n_{i}} x_{0}, T^{n_{i}+1} x_{0}, t\right) \geq M\left(x_{*}, x_{*}, t\right)=1
$$

and

$$
\lim _{i} \sup N\left(T^{n_{i}} x_{0}, T^{n_{i}+1} x_{0}, t\right) \leq N\left(x_{*}, x_{*}, t\right)=0, \forall t>0 .
$$

So $\lim _{i} M\left(T^{n_{i}} x_{0}, T^{n_{i}+1} x_{0}, t\right)=1$ and $\left.\lim _{i} N\left(T^{n_{i}} x_{0}, T^{n_{i}+1} x_{0}\right), t\right)=0, \forall t>0$. For any $n \in \mathbb{N}$ with $n>n_{1}$, there exists $n_{i}$ with $n_{i+1} \geq n>n_{i}$. From (3.11),

$$
\begin{aligned}
M\left(T^{n} x_{0}, x_{*}, t\right) \geq & M\left(T^{n-1} x_{0}, T^{n} x_{0}, t\right) * 1 * M\left(T^{n-1} x_{0}, x_{*}, t\right) \\
\geq & M\left(T^{n-1} x_{0}, T^{n} x_{0}, t\right) * M\left(T^{n-2} x_{0}, T^{n-1} x_{0}, t\right) \\
& * M\left(T^{n-2} x_{0}, x_{*}, t\right) \\
= & M\left(T^{n-2} x_{0}, T^{n-1} x_{0}, t\right) * M\left(T^{n-2} x_{0}, x_{*}, t\right) \\
\geq & \cdots \geq M\left(T^{n_{i}} x_{0}, T^{n_{i}+1} x_{0}, t\right) * M\left(T^{n_{i}} x_{0}, x_{*}, t\right)
\end{aligned}
$$


and

$$
\begin{aligned}
N\left(T^{n} x_{0}, x_{*}, t\right) \leq & N\left(T^{n-1} x_{0}, T^{n} x_{0}, t\right) \diamond 0 \diamond N\left(T^{n-1} x_{0}, x_{*}, t\right) \\
\leq & N\left(T^{n-1} x_{0}, T^{n} x_{0}, t\right) \diamond N\left(T^{n-2} x_{0}, T^{n-1} x_{0}, t\right) \\
& \diamond N\left(T^{n-2} x_{0}, x_{*}, t\right) \\
= & N\left(T^{n-2} x_{0}, T^{n-1} x_{0}, t\right) \diamond N\left(T^{n-2} x_{0}, x_{*}, t\right) \\
\leq & \cdots \leq N\left(T^{n_{i}} x_{0}, T^{n_{i}+1} x_{0}, t\right) \diamond N\left(T^{n_{i}} x_{0}, x_{*}, t\right) .
\end{aligned}
$$

Letting $n \rightarrow \infty\left(n_{i} \rightarrow \infty\right)$, we have

$$
\lim _{n} M\left(T^{n} x_{0}, x_{*}, t\right) \geq 1 \text { and } \lim _{n} N\left(T^{n} x_{0}, x_{*}, t\right) \leq 0, \forall t>0 .
$$

Hence we get $\lim _{n} T^{n} x_{0}=x_{*}$. This completes the proof.

Remark 4. Theorem 2 (i.e., Theorem 1 of [2]) is the immediate consequence of Theorem 4.

\section{Applications to product spaces}

In this chapter, we apply Theorem 3, Corollary 1 and Corollary 2 to obtain fixed point type theorems on the product of an I-FM space.

Theorem 5. Let $X$ be a complete I-FM space and $T: X \times X \rightarrow X$ such that be a mapping satisfying the following conditions:

(i) there exists $\left(x_{0}, y_{0}\right) \in X \times X$ such that

$$
\begin{aligned}
& \lim _{t \rightarrow \infty} M\left(\left(x_{0}, y_{0}\right), T^{i}\left(x_{0}, y_{0}\right), t\right)=1 \text { and } \\
& \lim _{t \rightarrow \infty} N\left(\left(x_{0}, y_{0}\right), T^{i}\left(x_{0}, y_{0}\right), t\right)=0, i=1,2, \ldots ;
\end{aligned}
$$

(ii) there exists a mapping $m: X \times X \rightarrow \mathbb{N}$ such that for any $(x, y),(u, v)$ in $X \times X$,

$$
\begin{aligned}
M\left(T^{m(x, y)}(x, y), T^{m(x, y)}(u, v), t\right) & \geq M((x, y),(u, v), \phi(t)), \\
N\left(T^{m(x, y)}(x, y), T^{m(x, y)}(u, v), t\right) & \leq N((x, y),(u, v), \phi(t)),
\end{aligned}
$$

where the function $\phi(t)$ satisfies condition $\left(\phi_{1}\right)$ and

$\left(\phi_{2}\right) \lim _{t \rightarrow \infty}[\phi(t)-t]=\infty$.

Then there exists exactly one point $q \in X$ such that $T^{m(q, y)}(q, y)=q$ for all $y \in X$ for each $m(q, y) \in \mathbb{N}$.

Proof. For a fixed $x \in X$ and $y=v$, the inequality (ii) corresponds to the condition (ii) of Theorem 3. Therefore for each $x \in X$, there exists one and only one $x(y)$ in $X$ such that $T^{m(x(y), y)}(x(y), y)=x(y)$ and $T^{m(x(y), y)}(x(v), v)=$ $x(v), m(x(y), y) \in \mathbb{N}$. 
Now, for every $y, v \in X$, from (ii) we get

$$
\begin{aligned}
M(x(y), x(v), t) & =M\left(T^{m(x(y), y)}(x(y), y), T^{m(x(y), y)}(x(v), v), t\right) \\
& \geq M(x(y), x(v), \phi(t)), \\
N(x(y), x(v), t) & =N\left(T^{m(x(y), y)}(x(y), y), T^{m(x(y), y)}(x(v), v), t\right) \\
& \leq N(x(y), x(v), \phi(t)) .
\end{aligned}
$$

On the other hand, as $M(x(y), x(v), t)$ is non-decreasing and $N(x(y), x(v), t)$ is non-increasing, we have

$$
\begin{aligned}
& M(x(y), x(v), t) \leq M(x(y), x(v), \phi(t)) \text { and } \\
& N(x(y), x(v), t) \geq N(x(y), x(v), \phi(t)) .
\end{aligned}
$$

Hence

$$
M(x(y), x(v), t)=M(x(y), x(v), \phi(t))=M\left(x(y), x(v), \phi^{n}(t)\right), \forall t>0,
$$
and

$$
N(x(y), x(v), t)=N(x(y), x(v), \phi(t))=N\left(x(y), x(v), \phi^{n}(t)\right), \forall t>0 .
$$

By the condition $\left(\phi_{1}\right)$,

$$
M(x(y), x(v), t)=1 \text { and } N(x(y), x(v), t)=0, \forall t>0 .
$$

Then by Definition $3[($ iii) and (ix)] we have $x(y)=x(v)$. So, $u(\cdot)$ is some constant $q \in X$ and conclusions of the theorem are obtained.

If $\phi(t)=\frac{t}{k}$ in Theorem 5 , we obtain an application on the product of an I-FM space of Corollary 1.

Corollary 3. Let $X$ be a complete $I-F M$ space and $T: X \times X \rightarrow X$ such that be a mapping satisfying the following conditions:

(i) there exists $\left(x_{0}, y_{0}\right) \in X \times X$ such that

$$
\begin{aligned}
& \lim _{t \rightarrow \infty} M\left(\left(x_{0}, y_{0}\right), T^{i}\left(x_{0}, y_{0}\right), t\right)=1 \text { and } \\
& \lim _{t \rightarrow \infty} N\left(\left(x_{0}, y_{0}\right), T^{i}\left(x_{0}, y_{0}\right), t\right)=0, i=1,2, \ldots ;
\end{aligned}
$$

(ii) there exists a mapping $m: X \times X \rightarrow \mathbb{N}$ such that for any $(x, y),(u, v)$ in $X \times X$,

$$
\begin{aligned}
& M\left(T^{m(x, y)}(x, y), T^{m(x, y)}(u, v), t\right) \geq M\left((x, y),(u, v), \frac{t}{k}\right), \\
& N\left(T^{m(x, y)}(x, y), T^{m(x, y)}(u, v), t\right) \leq N\left((x, y),(u, v), \frac{t}{k}\right),
\end{aligned}
$$

where $0<k<1$. Then the conclusion of Theorem 5 remains true.

Proof. Taking $\phi(t)=\frac{t}{k}$. Obviously, $\phi(t)$ satisfies the conditions $\left(\phi_{1}\right)$ and $\left(\phi_{2}\right)$. Therefore the conclusion follows from Theorem 5 directly. 
Corollary 4. Let $X$ be a complete $I-F M$ space and $T: X \times X \rightarrow X$ be a mapping. If there exists a mapping $m: X \times X \rightarrow \mathbb{N}$ such that for any $(x, y)$ in $X$,

$$
\begin{aligned}
M\left(T^{m(x, y)}(x, y), T^{m(x, y)}(u, v), t\right) & \geq M((x, y),(u, v), \phi(t)), \\
N\left(T^{m(x, y)}(x, y), T^{m(x, y)}(u, v), t\right) & \leq N((x, y),(u, v), \phi(t)),
\end{aligned}
$$

where the function $\phi(t)$ satisfies conditions $\left(\phi_{1}\right)$ and $\left(\phi_{2}\right)$. Then there exists exactly one point $q \in X$ such that $T^{m(q, y)}(q, y)=q$ for all $y \in X$ for each $m(q, y) \in \mathbb{N}$.

Proof. It is clear from proof of Theorem 5 .

Remark 5. Taking $\phi(t)=\frac{t}{k}(0<k<1)$ and $m(x, y) \equiv 1$ in Corollary 4, we obtain an application on product space of Theorem 1.

Conclusion. Essentially, from (2.4) it is easy to see that $T$ is continuous and (3.11) hold for any $x_{0} \in X$. In addition, from the compactness of $X$, $\left\{T^{n} x_{0}\right\}$ has an accumulation point. Hence Theorem 2 follows immediately from Theorem 4. Thus we improve and generalize the well-known fixed point theorems of Banach [4] and Edelstein [8] were given by Alaca et al. [2] in intuitionistic fuzzy metric spaces. Our main results are intuitionistic fuzzy version of Fang's results [10]. These fixed point theorems are applied to obtain solutions of fixed point type equations on product spaces.

Acknowledgement. The author would like to express their sincere thanks to Editor-in-Chief Professor Yeol Je Cho and the referees for their help in the improvement of this paper.

\section{References}

[1] C. Alaca and H. Efe, On Intuitionistic fuzzy Banach spaces, Int. J. Pure Appl. Math. 32 (2006), 347-364.

[2] C. Alaca, D. Turkoglu, and C. Yildiz, Fixed points in intuitionistic fuzzy metric spaces, Chaos, Solitons \& Fractals 29 (2006), 1073-1078.

[3] K. Atanassov, Intuitionistic fuzzy sets, Fuzzy Sets and Systems 20 (1986), 87-96.

[4] S. Banach, Theorie les operations Lineaires, Manograie Mathematyezne Warsaw Poland, 1932.

[5] S. S. Chang, Fixed point theorem of mappings on probabilistic metric spaces with applications, Sci. Sinica (Ser. A) 26 (1983), 1144-1155.

[6] D. Çoker, An introduction to intuitionistic fuzzy topological spaces, Fuzzy Sets and Systems 88 (1997), 81-89.

[7] Zi-Ke Deng, Fuzzy pseudo-metric spaces, J. Math. Anal. Appl. 86 (1982), 74-95.

[8] M. Edelstein, On fixed and periodic points under contractive mappings, J. London Math. Soc. 37 (1962), 74-79.

[9] M. A. Erceg, Metric spaces in fuzzy set theory, J. Math. Anal. Appl. 69 (1979), 205-230.

[10] Jin-Xuan Fang, On fixed point theorems in fuzzy metric spaces, Fuzzy Sets and Systems 46 (1992), 107-113.

[11] M. Grabiec, Fixed points in fuzzy metric spaces, Fuzzy Sets and Systems 27 (1988), $385-389$. 
[12] V. Gregori, S. Romaguera, and P. Veeramani, A note on intuitionistic fuzzy metric spaces, Chaos, Solitons \& Fractals 28 (2006), 902-905.

[13] G. Jungck, Commuting maps and fixed points, Amer. Math. Monthly 83 (1976), 261263.

[14] O. Kaleva and S. Seikkala, On fuzzy metric spaces, Fuzzy Sets and Systems 12 (1984), $225-229$.

[15] O. Kramosil and J. Michalek, Fuzzy metric and statistical metric spaces, Kybernetica 11 (1975), 326-334.

[16] R. Lowen, Fuzzy set theory, Kluwer Academic Pub., Dordrecht, 1996.

[17] K. Menger, Statistical metrics, Proc. Nat. Acad. Sci. USA 28 (1942), 535-537.

[18] S. N. Mishra, S. L. Singh, and V. Chadha, Coincidences and fixed points in fuzzy metric spaces, J. Fuzzy Math. 6 (1998), 491-500.

[19] R. P. Pant, Common fixed points of noncommuting mappings, J. Math. Anal. Appl. 188 (1994), 436-440.

[20] J. H. Park, Intuitionistic fuzzy metric spaces, Chaos, Solitons \& Fractals 22 (2004), 1039-1046.

[21] J. S. Park, Y. C. Kwun, and J. H. Park, A fixed point theorem in the intuitionistic fuzzy metric spaces, Far East J. Math. Sci. 16 (2005), no. 2, 137-149.

[22] A. Razani, Existence of fixed point for the nonexpansive mappings in intuitionistic fuzzy metric spaces, Chaos, Solitons \& Fractals 30 (2006), 367-373.

[23] R. Saadati and J. H. Park, On the intuitionistic topological spaces, Chaos, Solitons \& Fractals 27 (2006), 331-344.

[24] B. Schweizer and A. Sklar, Statistical metric spaces, Pacific J. Math. 10 (1960), 314-334.

[25] S. L. Singh and A. Tomar, Fixed point theorems in FM-spaces, J. Fuzzy Math. 12 (2004), 845-859.

[26] D. Turkoglu, C. Alaca, Y. J. Cho, and C. Yildiz, Common fixed point theorems in intuitionistic fuzzy metric spaces, J. Appl. Math. \& Computing 22 (2006), 411-424.

[27] D. Turkoglu, C. Alaca, and C. Yildiz, Compatible maps and compatible maps of types $(\alpha)$ and $(\beta)$ in intuitionistic fuzzy metric spaces, Demonstratio Mathematica 39 (2006), 671-684.

[28] L. A. Zadeh, Fuzzy sets, Inform. and Control 8 (1965), 338-353.

Department of Mathematics

FACUlty of Science And Arts

SINOP UNIVERSITY

57000 SINOP, TURKEY

E-mail address: cihangiralaca@yahoo.com.tr 